Bull. Austral. Math. Soc.

18D20, 18C99

VOL. 54 (1996) [489-501]

\title{
ENRICHED ACCESSIBLE CATEGORIES
}

Francis Borceux and Carmen Quinteriro

\begin{abstract}
We consider category theory enriched in a locally finitely presentable symmetric monoidal closed category $\mathcal{V}$. We define the $\mathcal{V}$-filtered colimits as those colimits weighted by a $\mathcal{V}$-flat presheaf and consider the corresponding notion of $\mathcal{V}$-accessible category. We prove that $\mathcal{V}$-accessible categories coincide with the categories of $\mathcal{V}$-flat presheaves and also with the categories of $\mathcal{V}$-points of the categories of $\mathcal{V}$-presheaves. Moreover, the $\mathcal{V}$-locally finitely presentable categories are exactly the $\mathcal{V}$-cocomplete finitely accessible ones. To prove this last result, we show that the Cauchy completion of a small $\mathcal{V}$-category $\mathcal{C}$ is equivalent to the category of $\mathcal{V}$-finitely presentable $\mathcal{V}$-flat presheaves on $\mathcal{C}$.
\end{abstract}

\section{INTRODUCTION}

The notions of locally finitely presentable category (see [3]) and symmetric monoidal closed category (see [2]) are now classical. Following a work of Kelly (see [6]) we fix a "locally finitely presentable symmetric monoidal closed category $\mathcal{V}$ ", meaning by this a category having all those properties and in which, moreover, it is assumed that a finite tensor product of finitely presentable objects is again finitely presentable (that is, the unit $I$ of the tensor product is finitely presentable and when $V, W$ are finitely presentable, so is $V \otimes W$ ). Observe that the categories of sets, Abelian groups, modules on a commutative ring, small categories, and all toposes of presheaves are instances of such categories $\mathcal{V}$. This fixes the assumptions on our base category $\mathcal{V}$; we shall no longer mention them in the rest of the paper.

In this paper, all categories, functors, limits, colimits and so on, will almost always be enriched over $\mathcal{V}$. For that reason and for the sake of brevity, we shall always omit the prefix $\mathcal{V}$-. We prefer using the term "ordinary" to emphasise, when some confusion could occur, the difference between a $\mathcal{V}$-structure and the ordinary underlying structure.

By a finite category is meant a category $\mathcal{D}$ with finitely many objects and such that each $\mathcal{D}(X, Y) \in \mathcal{V}$ is finitely presentable. A finite indexing type is a functor $F: \mathcal{D} \longrightarrow \mathcal{V}$ defined on a finite category $\mathcal{D}$ and such that each $F(D) \in \mathcal{V}$ is finitely presentable. A finite limit is a limit weighted by a finite indexing type; a finite colimit is a colimit

Received 4th January, 1996

Support for the first author by NATO grant CRG 941330 and FNRS grant 1.5.001.96F are acknowledged.

Copyright Clearance Centre, Inc. Serial-fee code: 0004-9729/96 \$A2.00+0.00. 
weighted by a contravariant finite indexing type (see [6]). In particular, cotensoring with a finitely presentable object is a finite limit process. A functor $F: \mathcal{A} \longrightarrow \mathcal{B}$ between finitely complete categories is left exact when it preserves finite limits. A presheaf $F: \mathcal{C}^{*} \longrightarrow \mathcal{V}$ is flat when its left Kan extension along the Yoneda embedding

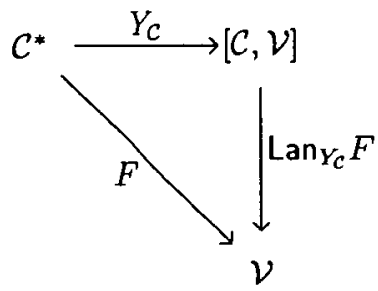

is left exact (see [6]).

In the ordinary case $\mathcal{V}=$ Set of the category of sets, the colimit of a functor $F$ weighted by a presheaf $G$

$$
\text { Set } \stackrel{G}{\longleftarrow} \mathcal{D}^{*}, \quad \mathcal{D} \stackrel{F}{\longrightarrow} \mathcal{C}
$$

coincides with the conical colimit of the composite

$$
\operatorname{Elt}(G) \stackrel{\phi_{G}}{\longrightarrow} \mathcal{D} \stackrel{F}{\longrightarrow} \mathcal{C}
$$

where $\operatorname{Elt}(G)$ is the category of elements of $G$ and $\phi_{G}$ is the obvious forgetful functor (see [1, II-6.6]). This conical colimit is filtered, that is the category $\operatorname{Elt}(G)$ is filtered, precisely when the presheaf $G$ is flat (see [1, I-6.3.1]). In the case of a general $\mathcal{V}$, it is well-known that the correct generalisation of the notion of limit is "weighted limit". Therefore the correct generalisation of the notion of filtered colimit should be "colimit weighted by a flat functor".

Definition 1.1: A filtered colimit is the colimit of some functor $F: \mathcal{D} \rightarrow \mathcal{C}$ weighted by a flat presheaf $G: \mathcal{D}^{*} \longrightarrow \mathcal{V}$.

We shall use the notation of [5] and write $G * F$ for the colimit of $F$ weighted by $G$. Analogously, $\{H, F\}$ will indicate the limit of $F$ weighted by a covariant presheaf $\boldsymbol{H}$.

Our Lemmas (1.5), (1.6) and (1.7) indicate that the previous definition generalises the notion of conical filtered colimit used in [6], while still recapturing the classical definition of filtered colimit in the case $\mathcal{V}=$ Set .

The objective of this paper is to generalise to the $\mathcal{V}$-case the theory of ordinary finitely accessible categories, using (1.1) as the notion of filtered colimit.

Definition 1.2: Let $\mathcal{A}$ be a category with filtered colimits. An object $A \in \mathcal{A}$ is finitely presentable when the representable functor $\mathcal{A}(A,-): \mathcal{A} \longrightarrow \mathcal{V}$ preserves filtered colimits. 
The definition of finitely presentable object used in [6] is different from ours, since it refers only to conical filtered colimits. But in [6] only finitely presentable categories are studied and in this particular case, we show in Corollary (2.3) that both definitions of finitely presentable object coincide. In the more general context of accessible categories, it is our definition (1.2) which allows us to generalise the well-known results of the case $\mathcal{V}=$ Set .

Definition 1.3: A category $\mathcal{A}$ is finitely accessible when

(1) $\mathcal{A}$ has filtered colimits;

(2) there exists a family $\left(C_{i}\right)_{i \in I}$ of finitely presentable objects of $\mathcal{A}$ such that every object $A \in \mathcal{A}$ can be written as a filtered colimit $A=G * F$ of a diagram

$$
\mathcal{V} \stackrel{G}{\longleftarrow} \mathcal{D}^{*}, \quad \mathcal{D} \stackrel{F}{\longrightarrow} \mathcal{C} \hookrightarrow \mathcal{A}
$$

in the full subcategory $\mathcal{C} \subseteq \mathcal{A}$ spanned by the objects $\left(C_{i}\right)_{i \in I}$.

Our main theorem generalises a well-known result in the case $\mathcal{V}=$ Set.

ThEOREM 1.4. For a category $\mathcal{A}$, the following conditions are equivalent:

(1) $\mathcal{A}$ is finitely accessible;

(2) $\mathcal{A}$ is equivalent to the category Flat $\left[\mathcal{C}^{*}, \mathcal{V}\right]$ of flat presheaves on a small category $\mathcal{C}$;

(3) $\mathcal{A}$ is equivalent to the category of points of a category $[\mathcal{C}, \mathcal{V}]$ of covariant presheaves, for some small category $\mathcal{C}$,

where as usual, a "point" of a finitely complete category $\mathcal{X}$ is a left exact left adjoint functor $\mathcal{X} \longrightarrow \mathcal{V}$.

The theorem is proved by the conjunction of Theorem 2.4 and Proposition 4.2. In fact, given a finitely accessible category $\mathcal{A}$ and a small category $\mathcal{C}$ as in conditions (2) or (3) of the theorem, we prove that the full subcategory $\mathcal{A}_{f} \subseteq \mathcal{A}$ of finitely presentable objects is equivalent to the Cauchy completion of $\mathcal{C}$. By a result of Johnson (see [4]), $\mathcal{A}_{f}$ is equivalent to a small category and can therefore be chosen as the "canonical" category $\mathcal{C}$ in the previous theorem. An important consequence of this is our Corollary 3.6: the locally finitely presentable categories in the sense of Kelly (see [6]) are exactly the cocomplete finitely accessible categories in our sense.

Let us conclude this section with the lemmas indicating that our notion of filtered colimit generalises correctly the notion of conical filtered colimit.

LEMma 1.5. Every conical filtered colimit is filtered in the sense of Definition 1.1 .

ProOF: Let $\mathcal{D}$ be an ordinary filtered category and write $\widehat{\mathcal{D}}$ for the free $\mathcal{V}$-category on $\mathcal{D}$. We must prove that the functor $\Delta_{I}: \widehat{\mathcal{D}}^{*} \longrightarrow \mathcal{V}$, constant on $I$, is flat. But 
$\Delta_{I}=\Delta_{I} * Y$ where $Y$ is the Yoneda embedding of $\widehat{\mathcal{D}}^{*}$. Since $\Delta_{I} * Y$ is by definition the conical colimit of $Y$, we get that $\Delta_{I}$ is a conical filtered colimit of representable functors; therefore $\Delta_{I}$ is flat (see [6]).

LEmma 1.6. When $\mathcal{V}=$ Set, the following conditions are equivalent for a small category $\mathcal{D}$ :

(1) the category $\mathcal{D}^{*}$ is filtered;

(2) the functor $\Delta_{1}: \mathcal{D}^{*} \longrightarrow$ Set, constant on the singleton, is flat.

In particular, every conical filtered colimit is filtered in the sense of Definition (1.1), and conversely.

Proof: The category of elements of $\Delta_{1}$ is $\mathcal{D}$ itself, from which the equivalence of Conditons (1) and (2) follows by [1, I-6.3.1]. This implies the first half of the last statement, while the converse is given by Corollary 1.5 .

Lемма 1.7. In $\mathcal{V}$, finite limits commute with filtered colimits.

Proof: Consider a finite indexing type $G: \mathcal{D} \longrightarrow \mathcal{V}$, a flat functor (that is, a "filtered indexing type") $H: \mathcal{C}^{*} \longrightarrow \mathcal{V}$, with $\mathcal{C}$ small, and an arbitrary functor $F$ : $\mathcal{D} \otimes \mathcal{C} \longrightarrow \mathcal{V}$. We must prove that

$$
H(?) *\{G(-), F(?,-)\} \cong\{G(-), H(?) * F(?,-)\} .
$$

Since each object $G(D)$ is finitely presentable, the result follows at once from the preservation of the filtered colimit $H(?) * F(?, D)$ by the functor $[G(D),-]$.

\section{Categories of flat presheaves}

We fix $\mathcal{V}$ as in the introduction and consider moreover a small category $\mathcal{C}$. This notation will remain fixed through this section and will not always be recalled.

PROPOSITION 2.1. The category Flat $\left[\mathcal{C}^{*}, \mathcal{V}\right]$ of flat presheaves is stable in the category $\left[\mathcal{C}^{*}, \mathcal{V}\right]$ under filtered colimits.

Proof: Let us consider

$$
\mathcal{V} \stackrel{G}{\leftrightarrows} \mathcal{D}^{*}, \quad \mathcal{D} \stackrel{F}{\longrightarrow} \text { Flat }\left[\mathcal{C}^{*}, \mathcal{V}\right] \hookrightarrow\left[\mathcal{C}^{*}, \mathcal{V}\right]
$$

with $G$ flat, and compute the corresponding colimit $G * F$ in $\left[C^{*}, \mathcal{V}\right]$. We consider further the Kan extension

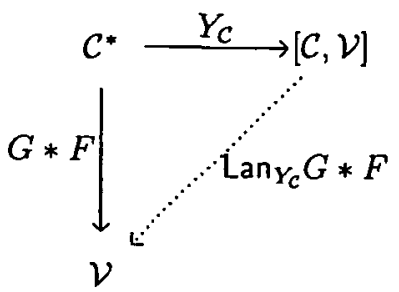


of $G * F$ along the Yoneda embedding and we must prove it is left exact. Given $H \in[\mathcal{C}, \mathcal{V}]$ and applying well-known identities (see [5, Chapter3])

$$
\begin{aligned}
\operatorname{Lan}_{Y_{c}}(G * F)(H) & \cong\left[Y_{\mathcal{C}}-, H\right] *(G * F) \\
& \cong H *(G * F) \\
& \cong(G * F) * H \\
& \cong G(?) *(F(?)(\bullet) * H(\bullet)) \\
& \cong((G(?) *-) \circ(F(?)(\bullet) *-))(H(\bullet))
\end{aligned}
$$

It suffices now to prove that both functors

$$
F(?) *-:[\mathcal{C}, \mathcal{V}] \longrightarrow[\mathcal{D}, \mathcal{V}], \quad G *-:[\mathcal{D}, \mathcal{V}] \longrightarrow \mathcal{V}
$$

where ? $\in \mathcal{D}$, are left exact. Since finite limits are computed pointwise in $[\mathcal{D}, \mathcal{V}]$, the first functor will be left exact if each

$$
F(D) *-:[\mathcal{C}, \mathcal{V}] \longrightarrow \mathcal{V}
$$

is left exact, which is the case since

$$
F(D) * H \cong H * F(D) \cong \operatorname{Lan}_{Y_{C}} F(D)(H)
$$

and $\operatorname{Lan}_{Y_{\mathcal{C}}} F(D)$ is exact because $F(D)$ is flat. Analogously, the functor $G *-\cong \operatorname{Lan}_{Y_{\mathcal{c}}} G$ is left exact because $G$ is flat.

Corollary 2.2. A presheaf $F: \mathcal{C}^{*} \longrightarrow \mathcal{V}$ is flat if and only if it is a filtered colimit of representable presheaves, that is, it has the form $F \cong G *\left(Y_{\mathcal{C}} \circ H\right)$ where

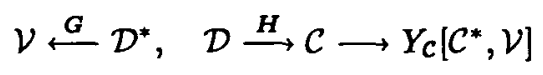

with $\mathcal{D}$ small and $G$ flat.

Proof: Every presheaf $F$ yields $F \cong F * Y_{C}$ (see $[5,(3.17)]$ ), from which the necessary condition follows. The converse implication follows from (2.1) and the flatness of representable functors (see [6]).

Corollary 2.3. Let $\mathcal{A}$ be a locally finitely presentable category in the sense of [6]. For an object $A \in \mathcal{A}$, the following conditions are equivalent

(1) $A$ is finitely presentable in the sense of Definition 1.3;

(2) $A$ is finitely presentable in the sense of [6]. 
Proof: By writing $\mathcal{A}_{K f} \subseteq \mathcal{A}$ for the full subcategory of finitely presentable objects in the sense of [6], and applying the results of that paper, we have the situation

$$
J: \mathcal{A} \longrightarrow \operatorname{Lex}\left[\mathcal{A}_{K f}^{*}, \mathcal{V}\right] \hookrightarrow\left[\mathcal{A}_{K f}^{*}, \mathcal{V}\right], \quad J(A)(B)=\mathcal{A}(B, A)
$$

where $J$ is an equivalence of categories and Lex indicates the category of left exact presheaves. Since $\mathcal{A}_{K f}^{*}$ is finitely cocomplete, left exact presheaves coincide with flat ones (see $[6,6.11])$. By Proposition $2.1, \operatorname{Lex}\left[\mathcal{A}_{K f}^{*}, \mathcal{V}\right]$ is therefore stable in $\left[\mathcal{A}_{K f}^{*}, \mathcal{V}\right]$ under filtered colimits in the sense of (1.1). This implies that in $\operatorname{Lex}\left[\mathcal{A}_{K f}^{*}, \mathcal{V}\right]$, filtered colimits are computed pointwise.

Now choose $A \in \mathcal{A}$ finitely presentable in the sense of Kelly, that is $A \in \mathcal{A}_{K f}$. It follows at once that $J(A)$ is the representable functor $\mathcal{A}_{K f}(-, A)$, and thus by the Yoneda lemma the representable functor

$$
[J(A),-]: \operatorname{Lex}\left[\mathcal{A}_{K f}^{*}, \mathcal{V}\right] \longrightarrow \mathcal{V}
$$

is evaluation at $A$. This functor preserves filtered colimits since these are pointwise in Lex $\left[\mathcal{A}_{K f}^{*}, \mathcal{V}\right]$. The converse follows at once from Lemma 1.5 .

We are now able to give a first characterisation of accessible categories.

THEOREM 2.4. For a category $\mathcal{A}$, the following conditions are equivalent:

(1) $\mathcal{A}$ is finitely accessible;

(2) $\mathcal{A}$ is equivalent to the category Flat $\left[C^{*}, \mathcal{V}\right]$ of flat presheaves on a small category $\mathcal{C}$.

ProOF: Assuming $\mathcal{A}$ finitely accessible and using the notation of Definition 1.3, write $\mathcal{C} \subseteq \mathcal{A}$ for the full subcategory generated by the objects $\left(C_{i}\right)_{i \in I}$. Each object $A \in \mathcal{A}$ can be written as a filtered colimit $A \cong G * F$

$$
\mathcal{V} \stackrel{G}{\longleftarrow} \mathcal{D}^{*}, \quad \mathcal{D} \stackrel{F}{\longrightarrow} \mathcal{C} \leftrightarrow \mathcal{A}
$$

of objects of $\mathcal{C}$. Since each object $C \in \mathcal{C}$ is finitely presentable and $F$ takes values in $\mathcal{C}$, we get

$$
\mathcal{A}(C, A) \cong \mathcal{A}(C, G * F) \cong G(-) * \mathcal{A}(C, F(-)) .
$$

Considering the functor

$$
Z: \mathcal{A} \longrightarrow\left[\mathcal{C}^{*}, \mathcal{V}\right], \quad Z(A)(C)=\mathcal{A}(C, A)
$$

we have just proved that $Z(A) \cong G *\left(Y_{\mathcal{C}} \circ F\right)$, where

$$
Y_{\mathcal{C}}: \mathcal{C}^{*} \longrightarrow[\mathcal{C}, \mathcal{V}], \quad C \mapsto \mathcal{C}(C,-)
$$


is the Yoneda embedding. Therefore $Z(A)$ is a filtered colimit of representable, thus flat, presheaves and is therefore flat by Proposition 2.1. But applying the criterion [6, 5.19.(iv)], our observations prove also that $\mathcal{C}$ is a dense subcategory of $\mathcal{A}$. Therefore the functor $Z$ is full and faithful, yielding already a full and faithful factorisation

$$
Z: \mathcal{A} \rightarrow \text { Flat }\left[\mathcal{C}^{*}, \mathcal{V}\right]
$$

It remains to prove that this factorisation $Z$ is essentially surjective on the objects.

A flat presheaf $F$ can be written as the filtered colimit $F \cong F * Y_{\mathcal{C}}$. Since $\mathcal{A}$ has filtered colimits, we define $A=F * i$, where $i: \mathcal{C} \hookrightarrow \mathcal{A}$ is the canonical inclusion. For each $C \in \mathcal{C}$,

$$
\begin{aligned}
Z(A)(C) & \cong \mathcal{A}(C, A) \cong \mathcal{A}(C, F * i) \\
& \cong F * \mathcal{A}(C, i) \cong F * \mathcal{C}(C,-) \cong F(C)
\end{aligned}
$$

implying easily that $Z(A) \cong F$.

Conversely, let us assume that $\mathcal{A}=$ Flat $\left[\mathcal{C}^{*}, \mathcal{V}\right]$ for some small $\mathcal{C}$. The representable functors are flat by (2.2), but they are also finitely presentable objects of $\mathcal{A}$. Indeed, if $G * F$

$$
\mathcal{V} \stackrel{G}{\leftrightarrows} \mathcal{D}^{*}, \quad \mathcal{D} \stackrel{F}{\longrightarrow} \mathcal{A}=\text { Flat }\left[\mathcal{C}^{*}, \mathcal{V}\right]
$$

is a filtered colimit of flat presheaves, by the Yoneda Lemma

$$
\mathcal{A}(\mathcal{C}(-, C), G * F) \cong(G * F)(C) \cong G * F(C) \cong G * \mathcal{A}(\mathcal{C}(-, C), F)
$$

since filtered colimits of flat functors are computed pointwise (see 2.1). One concludes by 2.2 .

\section{Categories of finitely presentable objects}

We fix $\mathcal{V}$ as in the introduction. We recall that a colimit $G * F$

$$
\mathcal{V} \stackrel{G}{\longleftarrow} \mathcal{C}^{*}, \quad \mathcal{C} \stackrel{F}{\longrightarrow} F \mathcal{A}
$$

is absolute when it is preserved by all possible functors $\mathcal{A} \longrightarrow \mathcal{B}$ for all possible $\mathcal{B}$. The indexing type $G$ is absolute when the colimit $G * F$ is absolute for all possible $\mathcal{A}$ and all possible $F$. It is proved in [8] that an indexing type $G$ is absolute when $G$, viewed as a profunctor $G: \mathcal{I} \rightarrow \mathcal{C}$ (with $\mathcal{I}$ the unit category) has a right adjoint profunctor. Moreover the category $\mathcal{A}$ is Cauchy complete precisely when it admits all colimits indexed by all absolute types. 
LEMmA 3.1. Every absolute indexing type $G: \mathcal{C}^{*} \longrightarrow \mathcal{V}$ is a flat functor.

ProOF: We must prove that the left Kan extension of $G$ along the Yoneda embedding

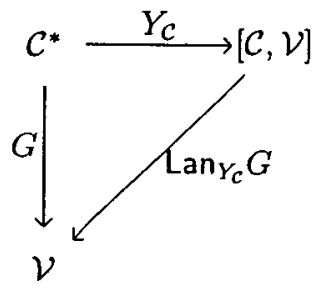

is left exact. For this choose a limit $\{F, H\}$

$$
\mathcal{V} \stackrel{F}{\longleftarrow} \mathcal{D} \stackrel{H}{\longrightarrow}[\mathcal{C}, \mathcal{V}]
$$

with $F$ a finite indexing type. Since

$$
\operatorname{Lan}_{Y_{c}} G(K) \cong K * G \cong G * K
$$

for $K \in[\mathcal{C}, \mathcal{V}]$, the thesis reduces to

$$
G *\{F, H\} \cong\{F(?), G * H(?)\}
$$

This relation is obtained by applying the functor $\{F,-\}$ to the absolute colimit $G * H$.

Our next goal is, in Theorem 2.4, to replace the small category $\mathcal{C}$ by the category $\mathcal{A}_{f} \subseteq \mathcal{A}$ of finitely presentable objects in $\mathcal{A}$. To achieve this, we prove first that this category is Cauchy complete, and next that it is actually the Cauchy completion of the small category $\mathcal{C}$ in $\mathbf{2 . 4}$.

Proposition 3.2. Every finite accessible category $\mathcal{A}$ is Cauchy complete.

Proof: Consider a colimit $G * F$

$$
\mathcal{V} \stackrel{G}{\longleftarrow} \mathcal{D}, \quad \mathcal{D} \stackrel{\boldsymbol{F}}{\longrightarrow} \mathcal{A}
$$

where $G$ is an absolute indexing type. By 3.1, the colimit $G * F$ is filtered, thus exists in $\mathcal{A}$. So $\mathcal{A}$ has all colimits with absolute indexing types and therefore is Cauchy complete (see [8]).

Corollary 3.3. If $\mathcal{A}$ is a finitely accessible category, the full subcategory $\mathcal{A}_{f} \subseteq \mathcal{A}$ of finitely presentable objects is Cauchy complete.

Proof: Consider the situation

$$
\mathcal{V} \stackrel{G}{\leftrightarrows} \mathcal{D}^{*}, \quad \mathcal{D} \stackrel{F}{\longrightarrow} \mathcal{A}_{f} \hookrightarrow \mathcal{A}
$$


with $G$ an absolute indexing type. The colimit $G * F$ exists in $\mathcal{A}$ by 3.2. Consider now a filtered colimit $K * H \in \mathcal{A}$

$$
\mathcal{V} \stackrel{K}{\longleftarrow} \mathcal{C}^{*}, \quad \mathcal{C} \stackrel{H}{\longrightarrow} \mathcal{A} .
$$

By 1.2, we must prove that

$$
\mathcal{A}(G * F, K * H) \cong K * \mathcal{A}(G * F, H) .
$$

Since the colimit $G * F$ is absolute, it is transformed in a limit by both contravariant functors $\mathcal{A}(-, K * H)$ and $K * \mathcal{A}(-, H)$. Therefore, since each $F(D)$ is finitely presentable and the colimit $K * H$ is filtered,

$$
\begin{aligned}
\mathcal{A}(G * F, K * H) & \cong\{G, \mathcal{A}(F, K * H)\} \\
& \cong\{G, K * \mathcal{A}(F, H)\} \cong K * \mathcal{A}(G * F, H)
\end{aligned}
$$

Proposition 3.4. The Cauchy completion of a small category $\mathcal{C}$ is equivalent to the full subcategory of finitely presentable objects in the category Flat $\left[\mathcal{C}^{*}, \mathcal{V}\right]$ of flat presheaves on $\mathcal{C}$. Up to an equivalence, this Cauchy completion is small.

Proof: For short, we write $\mathcal{A}=$ Flat $\left[\mathcal{C}^{*}, \mathcal{V}\right]$ and $\mathcal{A}_{f} \subseteq \mathcal{A}$ for the full subcategory of finitely presentable objects. By Corollary 2.2, the Yoneda embedding factors through $\mathcal{A}_{f}$ and by Corollary $3.3, \mathcal{A}_{f}$ contains the Cauchy completion $\overline{\mathcal{C}}$ of $\mathcal{C}$.

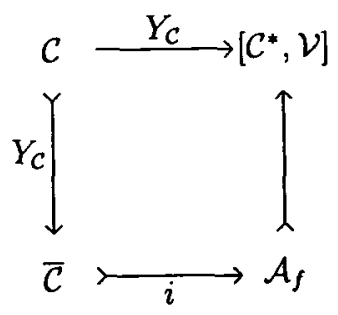

On the other hand, since $\mathcal{V}$ is locally finitely presentable, this Cauchy completion $\overline{\mathcal{C}}$ is equivalent to a small category, as proved in [4].

It remains to show that every object $F \in \mathcal{A}_{f}$ is in $\overline{\mathcal{C}}$. Since $F \cong F * Y_{\mathcal{C}}$, it suffices to show that the indexing type $F: \mathcal{C}^{*} \longrightarrow \mathcal{V}$ is absolute, that is, the existence of a right adjoint to $F: \mathcal{I} \rightarrow \mathcal{C}$, seen as a profunctor (see [8]).

It is well known (see [5]) that an object $F \in \mathcal{A}_{f}$ yields two profunctors

$$
F^{*}: \mathcal{I} \leftrightarrow \mathcal{A}_{f}, \quad F_{*}: \mathcal{A}_{f} \rightarrow \mathcal{I}
$$


with $\mathcal{I}$ the unit category and $F^{*} \dashv F_{*}$. As functors, $F^{*}$ and $F_{*}$ are those represented by $F$

$$
F^{*}=\mathcal{A}_{f}(-, F): \mathcal{A}_{f}^{*} \longrightarrow \mathcal{V}, \quad F_{*}=\mathcal{A}_{f}(F,-): \mathcal{A}_{f} \longrightarrow \mathcal{V} .
$$

It is useful to recall here the precise form of this adjunction.

$$
\begin{aligned}
1_{\mathcal{I}}(I, I) & =I \\
1_{\mathcal{A}_{f}}(H, K) & =\mathcal{A}_{f}(H, K) \\
\left(F_{*} \circ F^{*}\right)(I, I) & =\int^{H \in \mathcal{A}_{f}} F_{*}(H) \otimes F^{*}(H) \\
& =\int^{H \in \mathcal{A}_{f}} \mathcal{A}_{f}(F, H) \otimes \mathcal{A}_{f}(H, F) \\
& =\mathcal{A}_{f}(F, F) \\
\left(F^{*} \circ F_{*}\right)(H, K) & =F^{*}(H) \otimes F_{*}(K) \\
& =\mathcal{A}_{f}(H, F) \otimes \mathcal{A}_{f}(F, K) .
\end{aligned}
$$

The natural transformations of the adjunction are then

$$
\eta: 1_{\mathcal{I}} \Longrightarrow F_{*} \circ F^{*}, \quad \varepsilon: F^{*} \circ F_{*} \Longrightarrow 1_{\mathcal{A}_{f}}
$$

where $\eta_{I, I}$ is the identity on $F$ and $\varepsilon_{H, K}$ is composition. We know thus that $\eta$ and $\varepsilon$ satisfy the triangular identities for adjunction.

Restricting the previous situation to $\mathcal{C} \subseteq \mathcal{A}_{f}$ yields functors

$$
f^{*}: \mathcal{C}^{*} \stackrel{Y_{\mathcal{C}}^{*}}{\longrightarrow} \mathcal{A}_{f}^{*} \stackrel{F^{*}}{\longrightarrow} \mathcal{V}, \quad f_{*}: \mathcal{C} \stackrel{Y_{\mathcal{c}}}{\longrightarrow} \mathcal{A}_{f} \stackrel{F_{*}}{\longrightarrow} \mathcal{V}
$$

which we view as profunctors

$$
f^{*}: \mathcal{I} \rightarrow \mathcal{C}, \quad f_{*}: \mathcal{C} \rightarrow \mathcal{I} .
$$

By definition of $f^{*}$ and by the Yoneda lemma,

$$
f^{*}(C)=[\mathcal{C}(-, C), F] \cong F(C)
$$

so that the proof will be done if we prove that $f_{*}$ is right adjoint to $f^{*}$. The identity profunctor $1_{\mathcal{C}}: \mathcal{C} \rightarrow \mathcal{C}$ and the composite $f^{*} \circ f_{*}: \mathcal{C} \rightarrow \mathcal{C}$ are the restrictions to $\mathcal{C}$ of $1_{\mathcal{A}_{f}}$ and $F^{*} \circ F_{*}$, choosing $H$ and $K$ to be representable functors on $\mathcal{C}$. Therefore $\varepsilon$ restricts as a natural transformation $\varepsilon: f^{*} \circ f_{*} \Longrightarrow 1_{\mathcal{C}}$. If we can prove that $f_{*} \circ f^{*}=F_{*} \circ F^{*}$ as profunctors $\mathcal{I} \rightarrow \mathcal{I}$, then $\eta$ will also be a natural transformation $\eta: 1_{\mathcal{I}} \Longrightarrow f_{*} \circ f^{*}$ 
and the triangular identites for adjunction will hold for all objects in $\mathcal{C}$, since they do for all objects in $\mathcal{A}_{f}$.

Since $F$ is flat, the colimit $F \cong F * Y_{C}$ is filtered. Since $F$ is finitely presentable and observing that $f_{*}=\left[F, Y_{C}\right]$, we obtain

$$
[F, F] \cong\left[F, F * Y_{\mathcal{C}}\right] \cong F *\left[F, Y_{\mathcal{C}}\right] \cong f^{*} * f_{*} .
$$

We get then, by [5] (3.70),

$$
\left(f_{*} \circ f^{*}\right)(I, I) \cong \int^{C \in \mathcal{C}} f_{*}(C) \otimes f^{*}(C) \cong f_{*} \otimes f^{*} \cong f^{*} \otimes f_{*} \cong[F, F]
$$

which concludes the proof.

We are now ready to improve our Theorem 2.4.

Theorem 3.5. Every finitely accessible category $\mathcal{A}$ is equivalent to the category Flat $\left(\mathcal{A}_{f}^{*}, \mathcal{V}\right)$ of flat presheaves on the small full subcategory $\mathcal{A}_{f} \subseteq \mathcal{A}$ of finitely presentable objects.

Proof: We use the notation of Definition 1.3. It is obvious that every family of finitely presentable objects which contains the objects $\left(C_{i}\right)_{i \in I}$ satisfies a fortiori condition (2) of 1.3. Therefore, by 3.4 , there is no restriction in assuming that $\left(C_{i}\right)_{i \in I}$ is a representative set of all the finitely presentable objects in $\mathcal{A}$. But then the full subcategory $\mathcal{C} \subseteq \mathcal{A}$ generated by the $C_{i}$ 's is equivalent to $\mathcal{A}_{f}$ and we conclude the proof by Theorem 2.4 .

The following corollaries generalise other well-known properties in the case $\mathcal{V}=$ Set .

COROLlary 3.6. A finitely accessible category is locally finitely presentable if and only if it is cocomplete.

Proof: Let $\mathcal{A}$ be finitely accessible cocomplete and write $\mathcal{A}_{f}$ for the full subcategory of finitely presentable objects. Let us prove first that this category $\mathcal{A}_{f}$ is finitely cocomplete. For this consider

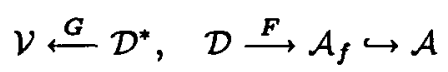

with $G$ a finite indexing type. The colimit $G * F$ exists in $\mathcal{A}$ by assumption. Consider now a filtered colimit $K * H \in \mathcal{A}$

$$
\mathcal{V} \stackrel{K}{\longleftarrow} \mathcal{C}^{*}, \quad \mathcal{C} \stackrel{H}{\longrightarrow} \mathcal{A}
$$

By 1.2, we must prove that

$$
\mathcal{A}(G * F, K * H) \cong K * \mathcal{A}(G * F, H) .
$$


This follows from the relations

$$
\begin{aligned}
\mathcal{A}(G * F, K * H) & \cong\{G(?), \mathcal{A}(F(?), K * H)\} \\
& \cong\{G(?), K * \mathcal{A}(F(?), H)\} \\
& \cong K *\{G(?), \mathcal{A}(F(?), H)\} \\
& \cong K * \mathcal{A}(G * F, H)
\end{aligned}
$$

which hold because the functor $\mathcal{A}(-, A)$ transforms colimits in limits, the functor $\mathcal{A}(F(?),-)$ preserves filtered colimits since $F$ takes values in $\mathcal{A}_{f}$, and finite limits commute in $\mathcal{V}$ with filtered colimits (see 1.7).

By Theorem 3.5 and [6, Theorem 6.11], one has now

$$
\mathcal{A} \cong \operatorname{Flat}\left[\mathcal{A}_{f}^{*}, \mathcal{V}\right] \cong \operatorname{Lex}\left[\mathcal{A}_{f}^{*}, \mathcal{V}\right]
$$

from which $\mathcal{A}$ is locally finitely presentable (see [6, Theorem 7.2]).

Conversely, a locally finitely presentable category $\mathcal{A}$ is finitely accessible and cocomplete by Corollary 2.3 and [6, Theorem 6.11].

COROLLARY 3.7. In a locally finitely presentable category, finite limits commute with filtered colimits.

Proof: If $\mathcal{C}$ is a small cocomplete category, finite limits in $\operatorname{Lex}\left[\mathcal{C}^{*}, \mathcal{V}\right]$ are computed pointwise (see [6]) as well as filtered colimits (see Proposition 2.1). One concludes the proof by Lemma 1.7.

\section{CATEgories OF POINTS}

We again fix $\mathcal{V}$ as in the introduction. The goal of this section is to generalise to the $\mathcal{V}$-context the classical characterisation of ordinary finitely accessible categories as categories of points of a topos of presheaves.

Definition 4.1: When $\mathcal{A}, \mathcal{B}$ are categories with finite limits, a geometric morphism $f: \mathcal{A} \longrightarrow \mathcal{B}$ is a pair of adjoint functors

$$
f_{*}: \mathcal{A} \longrightarrow \mathcal{B}, \quad f^{*}: \mathcal{B} \longrightarrow \mathcal{A}, \quad f^{*} \dashv f_{*},
$$

with $f^{*}$ left exact.

A point of $\mathcal{A}$ is a geometric morphism $f: \mathcal{V} \longrightarrow \mathcal{A}$. A morphism of points is a natural transformation between the corresponding left adjoint parts.

Proposition 4.2. For a category $\mathcal{A}$, the following conditions are equivalent:

(1) $\mathcal{A}$ is finitely accessible;

(2) $\mathcal{A}$ is the category of points of $[\mathcal{C}, \mathcal{V}]$ for a small category $\mathcal{C}$. 
Proof: Given a small category $\mathcal{C}$ and via Theorem 2.4 , it clearly suffices to prove the existence of an equivalence of categories

$$
\operatorname{Flat}\left[\mathcal{C}^{*}, \mathcal{V}\right] \simeq \operatorname{Point}[\mathcal{C}, \mathcal{V}]
$$

where "Point" indicates the category of points.

Theorem 5.33 in [5] implies that every cocontinuous (that is, small colimit preserving) functor $f^{*}:[\mathcal{C}, \mathcal{V}] \longrightarrow \mathcal{V}$ has a right adjoint. Thus the category Point $[\mathcal{C}, \mathcal{V}]$ is equivalent to the category of those functors $f^{*}:[\mathcal{C}, \mathcal{V}] \longrightarrow \mathcal{V}$ which preserve small colimits and finite limits. But [5, Theorem 4.51] implies that taking the left Kan extension along the Yoneda embedding induces an equivalence of categories

$$
\left[\mathcal{C}^{*}, \mathcal{V}\right] \simeq \operatorname{Cocont}([\mathcal{C}, \mathcal{V}], \mathcal{V})
$$

where "Cocont" indicates the category of cocontinuous functors. By definition of a flat functor and the previous observation on points, this equivalence restricts to the expected one.

\section{REFERENCES}

[1] F. Borceux, Handbook of categorical algebra I, II, III, Encyclopedia of Math. and its Appl. 50, 51,52 (Cambridge, 1994).

[2] S. Eilenberg and G.M. Kelly, 'Closed categories', in Proceedings Conference on Categorical Algebra, La Jolla 1965 (Springer-Verlag, Berlin, Heidelberg, New York, 1966), pp. 421-562.

[3] P. Gabriel and F. Ulmer, Lokal präsentierbare Kategorien, Springer Lecture Notes in Maths 221, 1971.

[4] S.R. Johnson, 'Small Cauchy completions', J. Pure Appl. Algebra 62 (1989), 35-45.

[5] G.M. Kelly, Basic concepts of enriched category theory, London Math. Society Lecture Note Series 64 (Cambridge University Press, Cambridge, 1982).

[6] G.M. Kelly, 'Structures defined by finite limits in the enriched context, I', Cahiers Topologie Géom. Différentielle Catégoriques XXIII-1 (1982), 3-42.

[7] R. Street, 'Absolute colimits in enriched categories', Cahiers Topologie Géom. Différentielle Catégoriques XXIV-4 (1983), 377-379.

Departement de Mathematiques

Universite de Louvain

2 Chemin du Cyclotron

1348 Louvain-la-Neuve

Belgium
Universidade de Vigo

Facultade de Ciencias Económicas e Empresariais

Lagoas - Marcosende s/n

36200 Vigo

Spain 